\title{
WWC3 inhibits epithelial-mesenchymal transition of lung cancer by activating Hippo-YAP signaling
}

This article was published in the following Dove Press journal: OncoTargets and Therapy

\author{
Qiang Han' \\ Joachim Kremerskothen ${ }^{2}$ \\ Xuyong Lin' \\ Xiupeng Zhang' \\ Xuezhu Rong' \\ Di Zhang' \\ Enhua Wang'
}

'Department of Pathology, College of Basic Medical Sciences, First Affiliated Hospital of China Medical University, Shenyang, People's Republic of China; ${ }^{2}$ Internal Medicine D, Department of Nephrology, Hypertension and Rheumatology, University Hospital Muenster, Muenster, Germany
Correspondence: Enhua Wang Department of Pathology, College of Basic Medical Sciences, First Affiliated Hospital of China Medical University, Shenyang, II0I22, People's Republic of China

$\mathrm{Tel}+86$ I338688 75 II

Fax +86 $242326 \quad 638$

Email wangeh@hotmail.com
Background: Though we recently reported that the WWC3 inhibits the invasiveness and metastasis of lung cancer by activating the Hippo pathway, the impact and underlying mechanisms of this process still remain unclear.

Methods: To identify the role of WWC3 in epithelial-mesenchymal transition of lung cancer, we performed immunohistochemistry to detect the expression levels of WWC3 and EMT-related biomarker, and analyzed their correlations in a cohort of 127 patients with NSCLC. Wound healing assay and cell invasion assay were applied to explore cell invasive ability change after WWC3 knockdown. qRT-PCR and immunoblotting were performed to assess mRNA and protein levels of EMT-related biomarkers and the main molecules changes of Hippo signaling caused by WWC3. Immunoprecipition was to examine WWC3 and LATS1 interaction.

Results: WWC3 knockdown drives a pronounced shift from the epithelial to the mesenchymal phenotype in lung cancer cells. In addition, WWC3 ectopic expression in lung cancer cells attenuates mesenchymal markers and increases the epithelial markers expressions; however, WWC3$\triangle W W$ plasmid abrogated these effects. WWC3 silencing by shRNA exerts the opposite effect. Furthermore, WWC3 levels were inversely correlated with the levels of EMT inducers (Snail and Slug) in lung cancer cells and specimens. Immunoblotting revealed that WWC3 wild-type upregulates large tumor suppressor (LATS1) and yes-associated protein (YAP) phosphorylation through its WW domain, hence activating Hippo pathway. Knockdown of YAP and LATS1, as well as the as the Verteporfin (VP) usage, could reverse this effect caused by WWC3 silencing.

Conclusion: These findings suggest that WWC3 works as a tumor suppressor to inhibit EMT process and confer its candidacy as a potential therapeutic target in lung cancer.

Keywords: WWC3, epithelial-mesenchymal transition, Hippo pathway, YAP, nonsmall-cell lung cancer

\section{Introduction}

Lung cancer is a common cancer that is the leading cause of cancer-related deaths worldwide, and its incidence remains increasing. Nonsmall-cell lung cancer (NSCLC) accounts for $80 \%$ of all lung cancer cases. ${ }^{1}$ Although three novel therapeutic modalities (surgical resection, chemotherapy, and radiotherapy) have been established, the longterm survival of lung cancer patients is still generally unsatisfactory. A variety of complex genetic, epigenetic, and microenvironmental factors play vital roles in the survival and malignant phenotype of tumor cells. The prognosis of patients with NSCLC correlates with overall tumor metastasis. ${ }^{1-4}$ Currently, some improvements in targeted therapy, including third-generation epidermal growth factor receptor (EGFR) tyrosine kinase inhibitors (TKIs) and immune checkpoint PD-1/PD-L1 blockade therapy, have gathered much attention; ${ }^{5-7}$ however, the acquired resistance to targeted therapy also limited its ability to prolong survival. The molecular carcinogenesis of lung cancer is 
characterized by multiple alterations in the gene expression, which lead to abnormal changes in signaling transduction pathways and biological behaviors. Thus, there is a need for new therapeutic targets and a better understanding of the mechanisms involved in the progression of NSCLC. ${ }^{8,9}$

The epithelial-mesenchymal transition (EMT) is a molecular process that allows epithelial cells to transform into a plastic and motile state with a mesenchymal phenotype. This characteristic is accompanied by modifications in adhesion, morphology, cellular architecture, migration potential, ${ }^{10,11}$ and altered expression of several genes involved in these processes. Several studies have suggested that the EMT process is critical for the invasion and metastasis of malignant tumors. ${ }^{12}$ However, the underlying mechanism has yet to be determined. Therefore, a comprehensive understanding of the molecular mechanisms of EMT remains vital for recently proposed precision medicine.

The Hippo pathway is an evolutionarily conserved signaling pathway that is important in a variety of biological processes, such as organismal development and cell growth and differentiation, and is implicated in the EMT process as well. ${ }^{13}$ In mammals, this pathway comprises a kinase cascade involving the Sav1/MST kinase complex and the MOB1/large tumor suppressor-1/large tumor suppressor-2 (LATS1/2) kinase complex that phosphorylates the transcription coactivators yes-associated protein (YAP) and TAZ. Once phosphorylated, YAP and TAZ are sequestered in the cytoplasm and undergo $\beta$-TrCP-mediated degradation. Upon inactivation of the Hippo pathway, stabilized YAP and TAZ translocate into the nucleus and bind to TEA domain transcription factors (TEAD) to activate target gene transcription. ${ }^{14}$ Verteporfin (VP), an US Food and Drug Administration-approved drug used in photodynamic therapy for macular degeneration, was recently identified as an inhibitor of YAP-TEAD binding. VP binds to YAP to change its conformation, thus abrogating its association with TEAD. ${ }^{15}$

We recently reported that $\mathrm{WW}$ and $\mathrm{C} 2$ domain containing protein-3 (WWC3), a homolog of the WWC (KIBRA/ WWC1, WWC2, and WWC3) gene family, interacts with LATS through its WW domain to activate the Hippo pathway, hence suppressing the invasiveness and metastasis of lung cancer. ${ }^{16}$ Hou et $a l^{17}$ demonstrated that WWC3 inhibits the proliferation and invasive ability of gastric cancer by stimulating the Hippo signaling activity. These results suggest that WWC3 is recognized as a tumor suppressor gene. However, the relationship between WWC3 and the EMT process remains unclear. Thus, in this study, we performed Western blot, immunohistochemistry (IHC), and immunofluorescence to explore the effect of WWC3 on EMT and its underlying mechanisms through dual regulation of WWC3 expression in lung cancer cells and provide a theoretical and experimental basis for lung cancer treatment strategies.

\section{Patients and methods}

\section{Patients and specimens}

We collected 127 tumor specimens, including NSCLC tissues and paired nontumor portions ( 32 cases; with $>5 \mathrm{~cm}$ distance from the primary tumor's edge), from patients (average age: 60 years) who underwent surgery at the First Affiliated Hospital of China Medical University from 2005 to 2014 after obtaining written informed consent from them and with the approval of the Institute Research Ethics Committee (No 2015 [LS]023, China Medical University). All tumor specimens were from the surgical resection. None of the patients had received chemotherapy or radiotherapy before tumor excision. According to the 2015 World Health Organization classification criteria of lung cancer, ${ }^{18} 73$ cases presented with adenocarcinoma and 54 cases presented with squamous cell carcinoma. According to the International Union of Cancer TNM staging standards in 2010, ${ }^{19} 99$ cases were in Phases I and II, whereas 28 cases were in Phase III.

\section{Immunohistochemistry}

Assays were performed as described previously. ${ }^{20}$ Briefly, the tissue sections were incubated with WWC3 rabbit polyclonal antibody (HPA039814, 1:200; Sigma-Aldrich Co., St Louis, MO, USA), rabbit monoclonal E-cadherin (3195, 1:200; Cell Signaling Technology, Danvers, MA, USA), rabbit monoclonal N-cadherin (13116, 1:200; Cell Signaling Technology), Snail1 goat polyclonal antibody (ab53519, 1:100; Abcam, Cambridge, MA, USA), and Slug rabbit polyclonal antibody (ab27568, 1:100; Abcam). The intensities of the WWC3, E-cadherin, N-cadherin, Snail1, and Slug stains were scored as follows: 0 (no staining), 1 (weak), 2 (moderate), and 3 (high). Percentage scores were assigned as follows: 1 (1\%-25\%), 2 (26\%-50\%), 3 (51\%-75\%), and $4(76 \%-100 \%)$. The scores of each tumor sample were multiplied to give a final score of $0-12$, and WWC3-positive expression was defined previously. ${ }^{16}$ For N-cadherin, Snail1, and Slug, tumor samples with scores $\geq 4$ were recognized as positive expression, scores between 1 and 4 were categorized as weak expression, and a score of 0 was considered as negative. The E-cadherin scores were determined by the percentage of membranous positive cells per slide, as in our previous study. ${ }^{21}$ PBS was used as the negative control.

\section{Cell cultures}

The human lung cancer cell lines, A549 and H1299, were obtained from the Shanghai Cell Bank (Shanghai, China) 
and authenticated by short tandem repeat DNA profiling. All cells were cultured in the Roswell Park Memorial Institute-1640 medium, $100 \mathrm{U} / \mathrm{mL}$ penicillin, and $100 \mu \mathrm{g} / \mathrm{mL}$ streptomycin (Sigma-Aldrich Co.) at $37^{\circ} \mathrm{C}$ and $5 \% \mathrm{CO}_{2}$ in a high humidity. VP (S1786) was purchased from Selleckchem (Shanghai, China).

\section{Plasmids and transfection}

Wild-type (WT) pEGFP-C2-WWC3, pEGFP-C2-WWC3$\Delta \mathrm{WW}$, and the corresponding pEGFP-C2 empty vectors were gifts from Dr Joachim Kremerskothen (University of Münster, Germany). pGPU6-shNC-RFP, pGPU6-shWWC31-RFP, and pGPU6-shWWC3-2-RFP were purchased from GenePharma (Shanghai, China). LATS1 siRNA (sc-35797), YAP siRNA (sc-38637), and control siRNA (sc-37007) were purchased from Santa Cruz Biotechnology Inc. (Dallas, TX, USA). Stable clonal cell lines were selected with $1,800 \mu \mathrm{g} / \mathrm{mL}$ of G418 (Thermo Fisher Scientific, Waltham, MA, USA) and were maintained at $900 \mu \mathrm{g} / \mathrm{mL}$ of G418. Lipofectamine 3000 (Thermo Fisher Scientific) transfection reagent was used for plasmid transfection.

\section{Western blot analysis}

Total protein from the cell lines was extracted with lysis buffer (Thermo Fisher Scientific) and quantified using the Bradford method. Sixty micrograms of protein were separated by sodium dodecyl sulfate polyacrylamide gel electrophoresis $(10 \%)$. After transfer, the polyvinylidene fluoride membranes (EMD Millipore, Billerica, MA, USA) were incubated overnight at $4{ }^{\circ} \mathrm{C}$ with the following antibodies: WWC3 $(1: 1,000$; Sigma-Aldrich Co.), glyceraldehyde-3-phosphate dehydrogenase (GAPDH; 1:1,000; Santa Cruz Biotechnology Inc.), E-cadherin (3195, 1:1,000), N-cadherin (13116, 1:1,000), ZEB1 (3396, 1:500), LATS1 (9153, 1:500), p-LATS1 (8654, 1:500), p-YAP (4911, 1:500), YAP (14074, 1:500), and p-YAP (4912, 1:500), all from Cell Signaling Technology, and Snail1 (ab53519, 1:500) and Slug (ab27568, 1:500), both from Abcam. After incubation with peroxidase-coupled antimouse immunoglobulin G (IgG) and anti-rabbit IgG (Santa Cruz Biotechnology Inc.) at $37^{\circ} \mathrm{C}$ for $2 \mathrm{~h}$, bound proteins were viewed using electro-chemi-luminescence (Thermo Fisher Scientific) and detected using BioImaging Systems (UVP Inc., Upland, CA, USA). The relative protein levels were calculated based on GAPDH as the loading control.

\section{Quantitative real-time polymerase chain reaction (PCR; SYBR Green method)}

Quantitative real-time PCR was performed using SYBR Green PCR Master Mix (Thermo Fisher Scientific) in a total volume of $20 \mu \mathrm{L}$ on the 7900 HT Fast Real-Time PCR System (Thermo Fisher Scientific) as follows: $95^{\circ} \mathrm{C}$ for $30 \mathrm{~s}$, 40 cycles at $95^{\circ} \mathrm{C}$ for $5 \mathrm{~s}$, and $60^{\circ} \mathrm{C}$ for $30 \mathrm{~s}$. A dissociation step was performed to generate a melting curve to confirm the specificity of the amplification. Beta-actin was used as the reference gene. The relative levels of gene expression were represented as $\Delta C_{t}=C_{t}$ gene $-C_{t}$ reference, and the fold change of the gene expression was calculated by the $2^{-\Delta \Delta C_{\mathrm{t}}}$ method. Experiments were repeated in triplicate. The primer sequences used are listed in Table 1.

\section{Immunofluorescence}

Cells were fixed with 4\% paraformaldehyde, blocked with 3\% bovine serum albumin, and incubated with WWC3 antibody (1:100; Sigma-Aldrich Co.), and Vimentin (5741, 1:100; Cell Signaling Technology) overnight at $4^{\circ} \mathrm{C}$, followed by fluorescein isothiocyanate-conjugated secondary antibodies, added at room temperature for $1 \mathrm{~h}$. Cells were counterstained with 4',6-diamidino-2-phenylindole. Epifluorescence microscopy was performed using an inverted Nikon TE300 microscope (Nikon Instruments, Melville, NY, USA), and confocal microscopy was performed using a Radiance 2000 laser scanning confocal microscope (Carl Zeiss Meditec AG, Jena, Germany).

\section{Wound healing assay}

Wounds were inflicted on the cells using a 200-mL pipette tip when the cultured cells reached a density of $<90 \%$ confluence. The cells were washed to remove debris before subculturing in a $2 \%$ serum culture medium. Wound healing within the scrape line was observed at different time points, and representative scrape lines for each cell line were photographed. Duplicate wells for each condition were examined for each experiment, and each experiment was repeated

Table I Primers for real-time qRT-PCR

\begin{tabular}{|c|c|}
\hline \multicolumn{2}{|c|}{ Primer sequences $\left(5^{\prime} \rightarrow 3^{\prime}\right)$} \\
\hline \multirow[t]{2}{*}{ WWC3 } & 5' CAAGAGCGCATGTTGAAGGAA 3' \\
\hline & 5' CGCTGCTGCTTAATCTGGTAGA 3' \\
\hline \multirow[t]{2}{*}{ Snail } & 5' AGTTTACCTTCCAGCAGCCCTAC 3' \\
\hline & 5' GCCTTTCCCACTGTCCTCATC 3' \\
\hline \multirow[t]{2}{*}{ Slug } & $5^{\prime}$ TGCCTGTCATACCACAACCAGA $3^{\prime}$ \\
\hline & 5' GGACTCACTCGCCCCAAAGA 3' \\
\hline \multirow[t]{2}{*}{$\mathrm{CDHI}$} & 5' CGAGAGCTACACGTTCACGG 3' \\
\hline & 5' GGGTGTCGAGGGAAAAATAGG 3' \\
\hline \multirow[t]{2}{*}{$\mathrm{CDH} 2$} & 5' TCAGGCGTCTGTAGAGGCTT 3' \\
\hline & $5^{\prime}$ ATGCACATCCTTCGATAAGACTG $3^{\prime}$ \\
\hline \multirow[t]{2}{*}{ Occludin } & 5' ACAAGCGGTTTTATCCAGAGTC $3^{\prime}$ \\
\hline & 5' GTCATCCACAGGCGAAGTTAAT 3' \\
\hline \multirow[t]{2}{*}{$\beta$-actin } & 5' ATAGCACAGCCTGGATAGCAACGTAC 3' \\
\hline & $5^{\prime}$ CACCTTCTACAATGAGCTGCGTGTG $3^{\prime}$ \\
\hline
\end{tabular}

Abbreviations: $q R T-P C R$, quantitative reverse transcription polymerase chain reaction; $\mathrm{WWC}, \mathrm{WW}$ and $\mathrm{C} 2$ domain containing protein-3; CDHI, E-cadherin; $\mathrm{CDH} 2, \mathrm{~N}$-cadherin. 
in triplicate. The distance of the wound was optically measured using ImageJ software (Bethesda, MD, USA).

\section{Transwell invasion assays}

Transwell plates (Costar; Corning Incorporated, Corning, NY, USA) precoated with Matrigel (BD Biosciences, San Jose, CA, USA) were used to perform the cell invasion assays. Briefly, the transwell plates were placed onto 24-well plates and a $500 \mathrm{~mL}$ of culture medium $(15 \%$ fetal bovine serum [FBS]) per well was added to the lower chamber as a chemoattractant. Next, $100 \mathrm{~mL}$ of the cells $\left(1 \times 10^{5}\right)$ suspended in the culture medium with $2 \% \mathrm{FBS}$ were added to the upper chamber. Cells in the chambers were incubated in a humidified incubator for $24 \mathrm{~h}$. Cells were fixed in methanol for $15 \mathrm{~min}$ and stained with hematoxylin for $10 \mathrm{~min}$. Five high power fields $(400 \times)$ were randomly selected in each chamber to observe the cells, and Cell Counter software (Hercules, CA, USA) was used to count the stained invading cells. Each experimental group was repeated three times.

\section{Statistical analysis}

All statistical analyses were performed using SPSS 22.0 (IBM Corporation, Armonk, NY, USA). The IHC results were analyzed using the chi-square test and Spearman's rank correlation. Differences between the groups were determined using Student's $t$-test. $P$-values $<0.05$ were considered as statistically significant.

\section{Results}

WWC3 knockdown induces EMT features and is correlated with malignancy in lung cancer cells

Deregulating the upstream Hippo pathway signaling molecules, such as Willin/FRMD6, induces the EMT; therefore, we examined the impact of WWC3 on the EMT process. A549 cells were expressed at the highest levels in the lung cancer cell lines we tested. ${ }^{16}$ We silenced WWC 3 expression using two independent short-hairpin RNAs (shRNAs) in the A549 cells (selected by G418). Interestingly, A549 cells with the WWC3 knockdown displayed a spindle-like morphology with a scattered distribution indicative of an EMT feature, whereas the control group retained a cobblestonelike appearance with tight cell-cell junctions (Figure 1A). The EMT process is closely related to tumor malignancy; ${ }^{22}$ therefore, we performed wound healing and transwell assays to examine the influence of WWC3 silencing on the migration and invasion of lung cancer cells. The results indicated that a knockdown of WWC3 enhanced the wound healing and invasive abilities of A549 cells compared with those of the control group (Figure 1B-E). This suggests a close relationship between WWC3 expression levels and EMT features and tumor malignancy.

\section{WWC3 levels correlate with biomarker expression in EMT processes}

The EMT process is often accompanied by the epithelial markers E-cadherin (CDH1), ZO-1, and occludin and a gain of the mesenchymal marker N-cadherin (CDH2). Therefore, we transfected a WT WWC3 plasmid and its mutant, deleting the WW domain in the H1299 cell line, which exhibits low WWC3 expression. ${ }^{16}$ Western blot analysis confirmed that ectopic WWC3 expression significantly upregulated E-cadherin, ZO-1, and occludin and downregulated $\mathrm{N}$-cadherin expression at both the protein and the mRNA levels; however, a lack of the WW domain abrogated this effect. Conversely, WWC3 knockdown using the two independent shRNAs had the opposite effect (Figure 2A-D). Immunofluorescence indicated that WWC3 silencing promoted the expression of N-cadherin (Figure 2E). IHC and statistical analysis showed that WWC3 is positively associated with E-cadherin expression in lung cancer specimens $(P=0.002)$ and is negatively correlated with $\mathrm{N}$-cadherin expression ( $P=0.022$; Figure $2 \mathrm{~F}$ and Table 2$)$. These results indicate that the WWC3 level correlates with biomarker expression in EMT processes.

\section{WWC3 negatively regulates EMT-inducing transcription factors Snail and Slug}

Some transcription factors, such as Snail, Slug, and ZEB1, are key regulators of the EMT process. We examined the impact of WWC 3 expression on these molecules. A Western blot analysis indicated that ectopic WT WWC3 expression significantly downregulated Snail and Slug, but not ZEB1, compared with the control group, and deleting the WW domain of WWC3 abrogated this effect. Conversely, WWC3 knockdown had the opposite effect (Figure 3A and B). These results were supported by quantitative reverse transcription polymerase chain reaction (qRT-PCR) analysis (Figure 3C). To confirm the relationship between WWC3 and Snail and Slug in lung cancer specimens, IHC, and statistical analysis were performed. The results showed that WWC3 negatively correlates with Snail $(P=0.001)$ and Slug $(P=0.012)$ expression (Figure 3D and Table 3). Collectively, our findings demonstrated that WWC3 negatively regulates the EMT-inducing transcription factors Snail and Slug. 


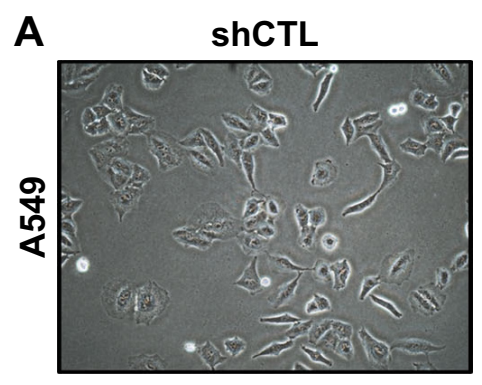

B
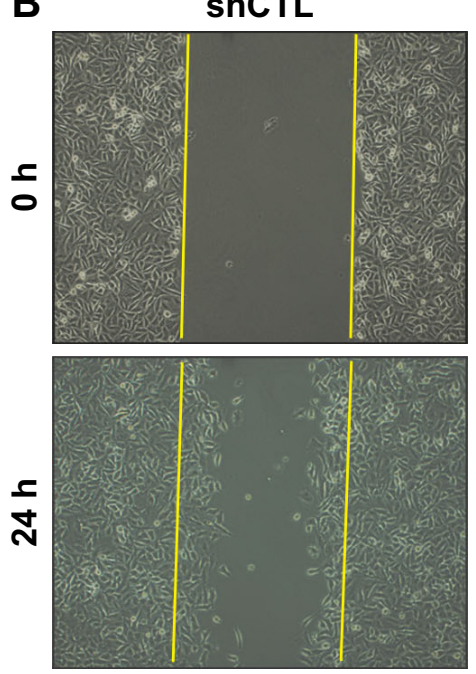

C

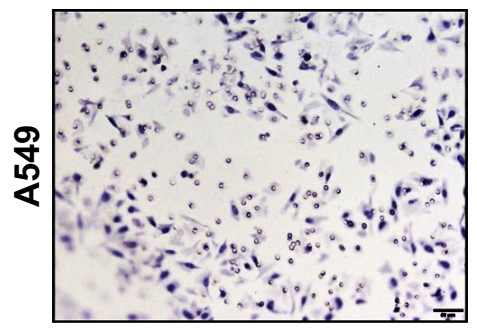

D

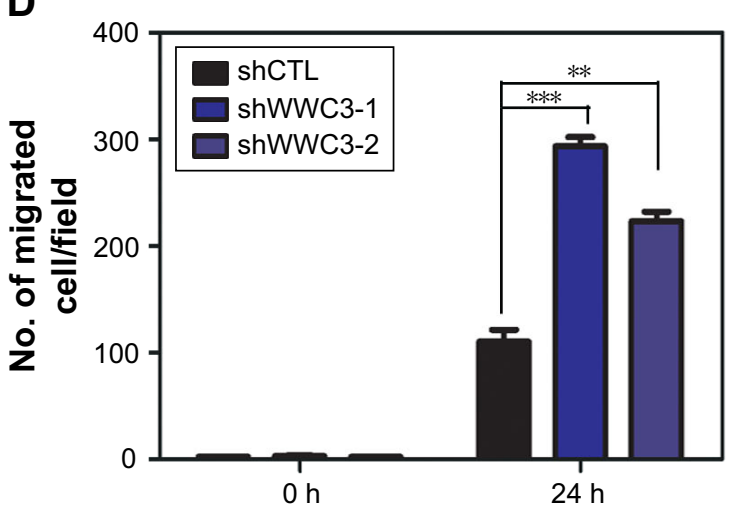

shWWC3-1

shWWC3-1

shWWC3-1
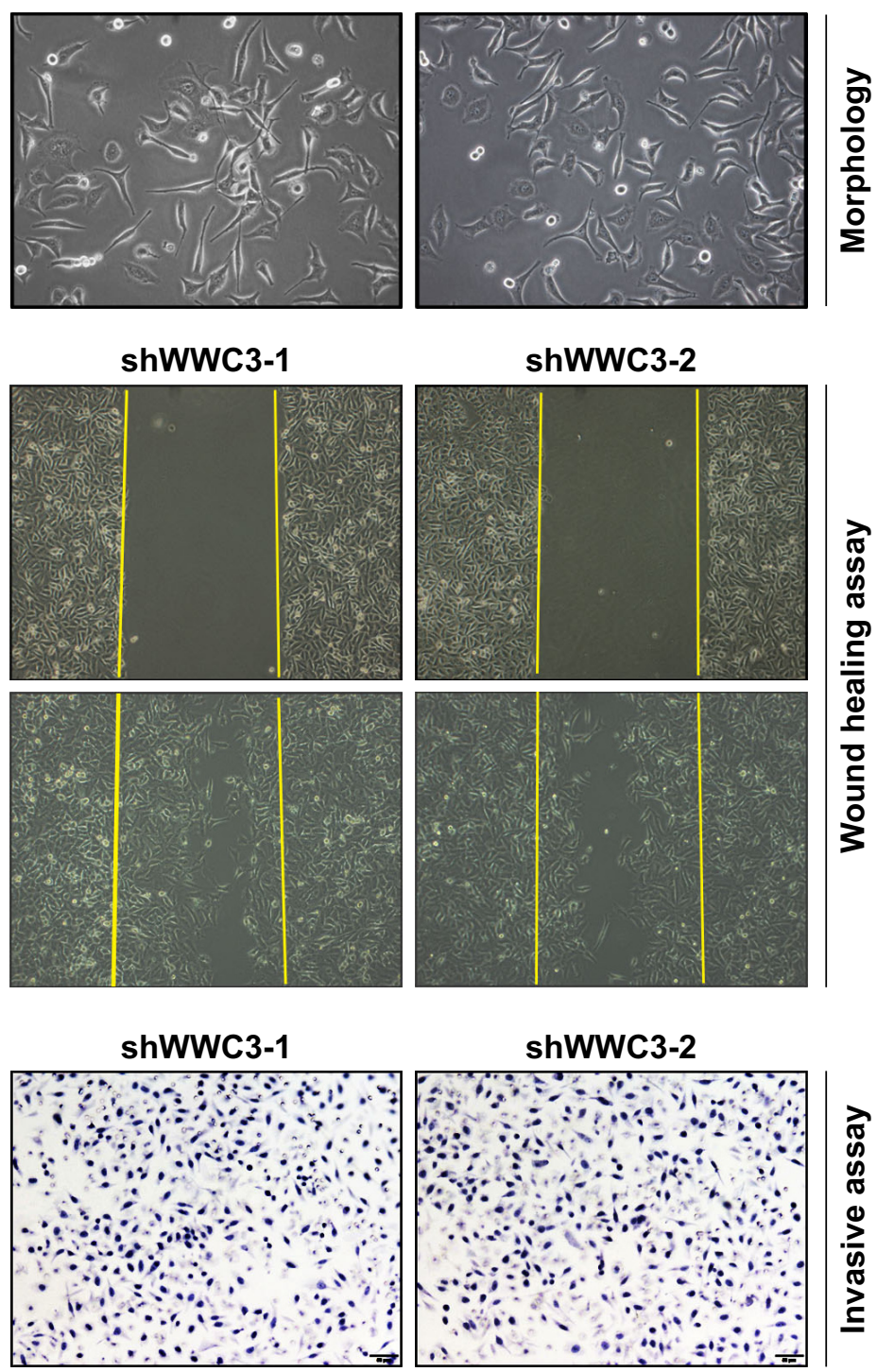

E

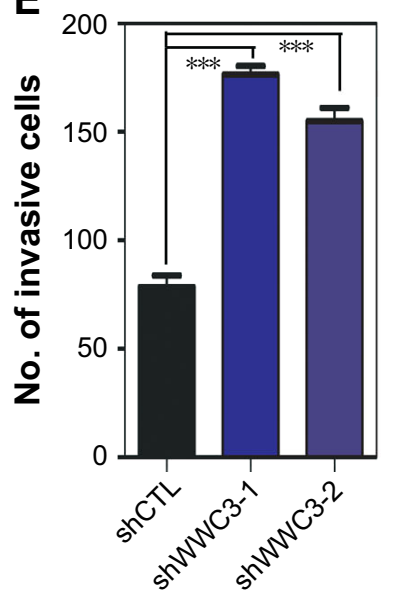

Figure I Knockdown of WWC3 induces the EMT and promotes the malignancy of lung cells.

Notes: (A) WWC3 silencing induces a morphology change in A549 cells. Representative phase-contrast images of cells growing in monolayer cultures and transfected with either shCTL or two independent shRNAs targeting WWC3 (WWC3-shRNA-I and -2). (B and D) Representative photographs of scratched areas of the confluent cell monolayer at 0 and $24 \mathrm{~h}$ after wounding with the cell migration indexes shown. All experiments were performed in triplicate. (C and $\mathbf{E})$ Boyden chamber transwell assay of cellular invasion and the mean number of cells in five fields per membrane. Data represent the mean $\pm S D$. $* * P<0.0 \mathrm{I}$ and $* * * P<0.00 \mathrm{I}$ by Student's $t$-test. The magnification is $400 \times$.

Abbreviations: WWC3, WW and C2 domain containing protein-3; EMT, epithelial-mesenchymal transition; shCTL, short-hairpin RNA control. 
A

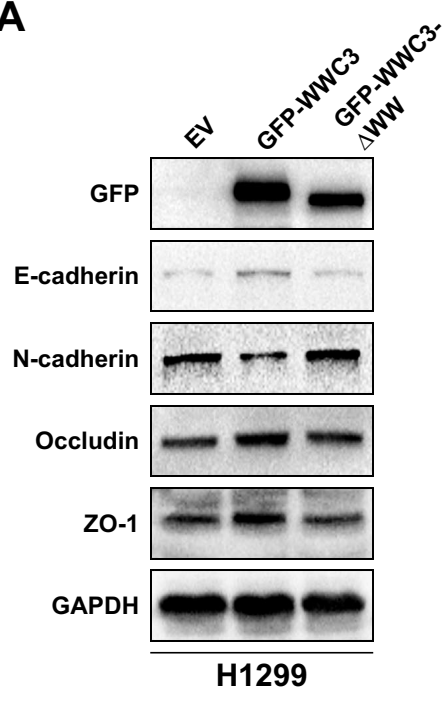

B

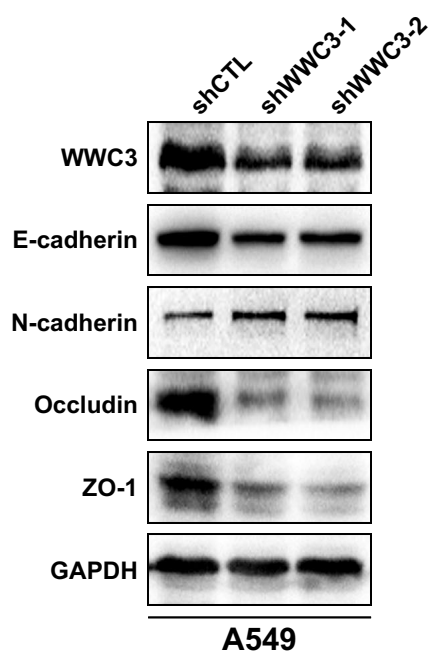

C

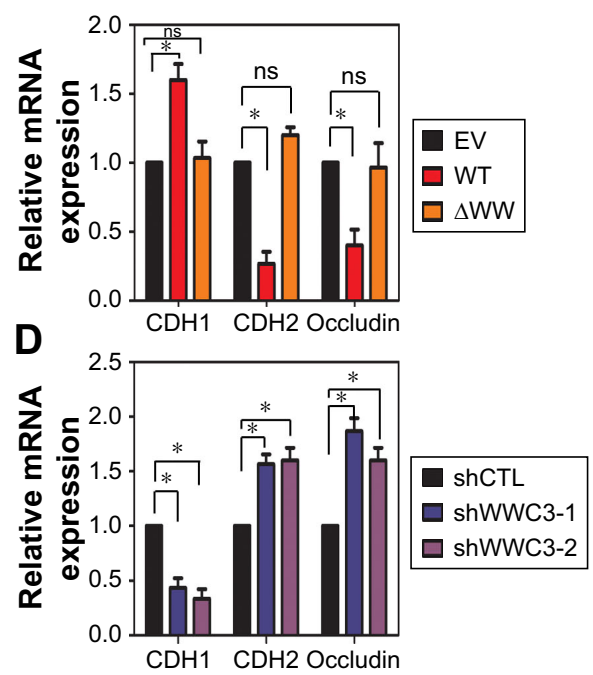

E

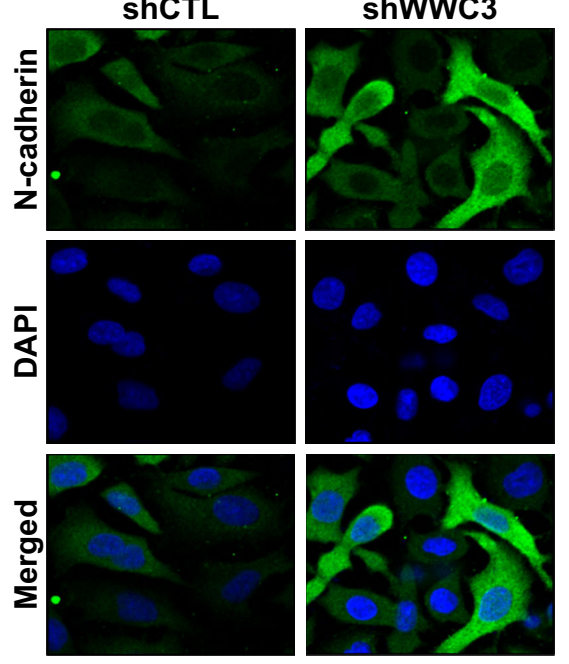

$\mathbf{F}$

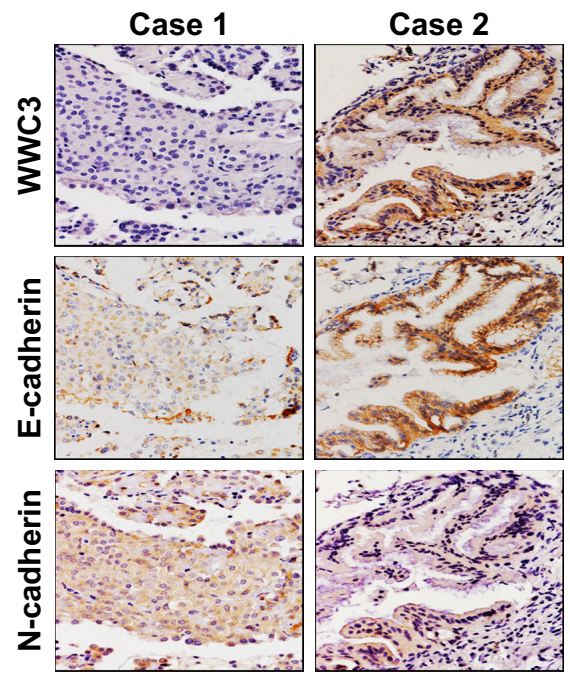

Figure 2 WWC3 level correlates with the expression of EMT process biomarkers.

Notes: (A and C) HI299 cells were transfected with WT WWC3 or mutant plasmid (- $\mathrm{WWW}$ ) and an EV, and $48 \mathrm{~h}$ later, cells were lysed and the proteins and mRNA were detected by immunoblotting and RT-qPCR, respectively. GAPDH expression served as a loading control. (B and D) Knockdown of WWC3 in A549 cells using two independent shRNAs had the opposite effect. (E) Silencing WWC3 enhanced N-cadherin expression. A549 cells were transfected with shRNA-WWC3 or shCTL for $48 \mathrm{~h}$ and then analyzed for endogenous $\mathrm{N}$-cadherin with confocal microscopy (magnification, 400x). (F) The representative photographs of the expression correlation between WWC3 and E-cadherin and N-cadherin in human lung cancer specimens (magnification, 400x). All experiments were performed in triplicate. Data represent the mean \pm SD. $* P<0.05$.

Abbreviations: WWC3, WW and C2 domain containing protein-3; EMT, epithelial-mesenchymal transition; WT, wild type; EV, empty vector; RT-qPCR, reverse transcription-quantitative polymerase chain reaction; GAPDH, glyceraldehyde-3-phosphate dehydrogenase; shCTL, short-hairpin RNA control; ns, nonsignificant; DAPI, 4',6-diamidino-2-phenylindole.

Table 2 Correlation of WWC3 with the expression of E-cadherin and N-cadherin in 127 NSCLC specimens

\begin{tabular}{|c|c|c|c|c|}
\hline \multirow[t]{2}{*}{ EMT-markers } & \multicolumn{2}{|l|}{ WWC3 } & \multirow[t]{2}{*}{$\chi^{2}$} & \multirow{2}{*}{$\begin{array}{l}\text { P-value } \\
\text { (two sided) }\end{array}$} \\
\hline & Negative & Positive & & \\
\hline E-cadherin & & & 10.182 & $0.002 *$ \\
\hline Negative & 25 & 6 & & \\
\hline Positive & 46 & 50 & & \\
\hline $\mathrm{N}$-cadherin & & & 6.060 & $0.022 *$ \\
\hline Negative & 54 & 31 & & \\
\hline Positive & 17 & 25 & & \\
\hline
\end{tabular}

Note: *Significant difference.

Abbreviations: WWC3, WW and C2 domain containing protein-3; NSCLC, nonsmall-cell lung cancer.

\section{WWC3 activates the Hippo pathway via} LATSI, suppressing EMT processes

Previous studies have confirmed that the dysregulation of multiple signaling transduction pathways, including the Hippo pathway, contributes to the EMT process. ${ }^{23}$ Recent reports indicate that YAP is imported into the nucleus to promote the transcription of Snail and Slug. ${ }^{24,25}$ In our previous work, we demonstrated that WWC3 can interact with LATS1 via its WW domain to promote the phosphorylation of LATS1 and YAP, thereby inhibiting YAP from being 

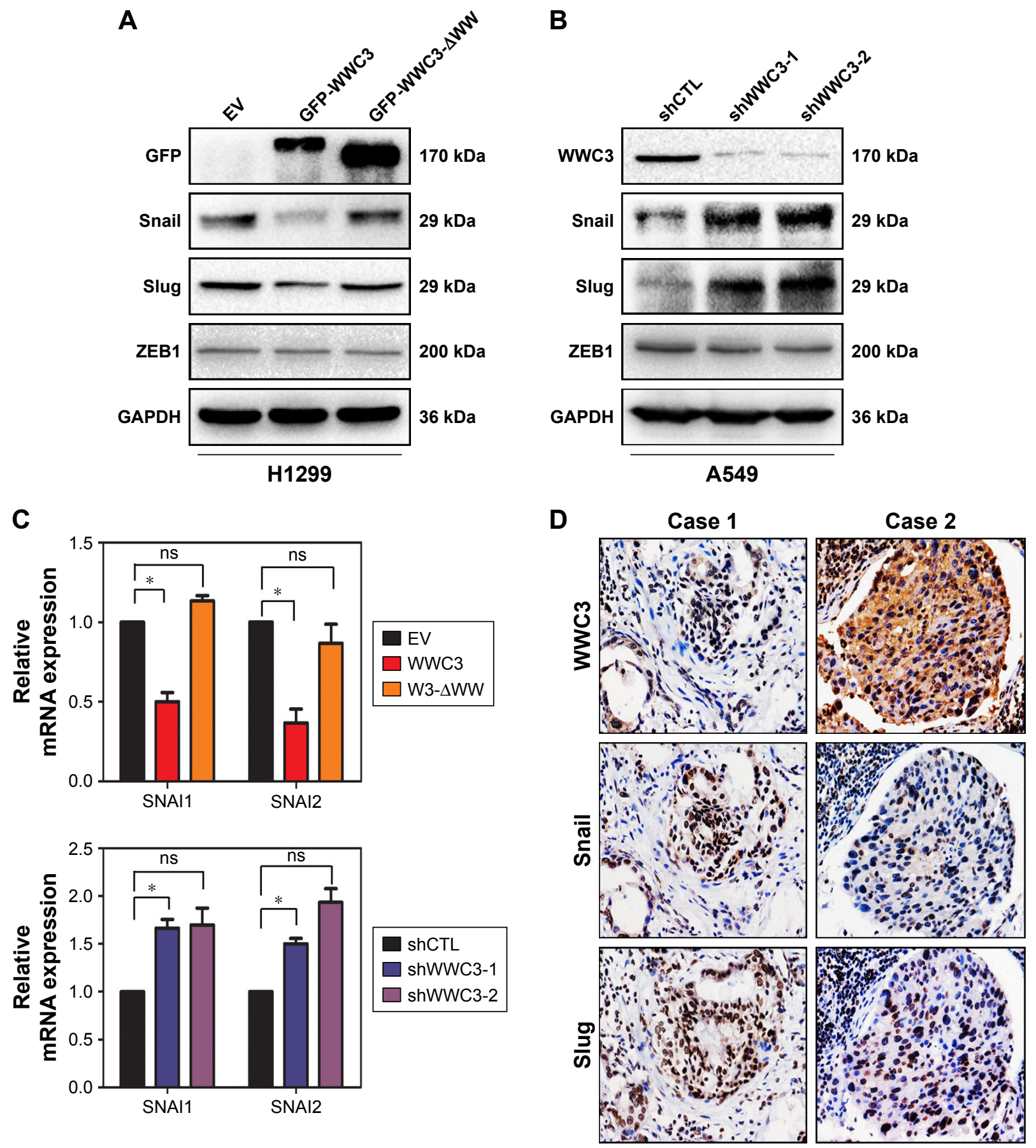

Figure 3 WWC3 negatively regulates EMT-inducing transcription factors Snail and Slug.

Notes: (A and C) WWC3 transfection in HI299 cells downregulated the protein and mRNA expression of EMT-related transcription factors Snail and Slug; WW domain deletion of WWC3 abrogated this effect. (B and C) WWC3 knockdown in A549 cells upregulated the expression of EMT-related transcription factors. GAPDH expression served as a control. (D) The representative photographs of the expression correlation between WWC3 and Snail and Slug in human lung cancer specimens (magnification, 400 $\times$ ). Data represent the mean \pm SD. $* P<0.05$.

Abbreviations: WWC3, WW and C2 domain containing protein-3; EMT, epithelial-mesenchymal transition; GAPDH, glyceraldehyde-3-phosphate dehydrogenase; $E V$, empty vector; shCTL, short-hairpin RNA control; ns, nonsignificant.

imported into the nucleus to activate the Hippo pathway. ${ }^{16}$ Thus, we assumed that WWC3 inhibits EMT features by activating the Hippo pathway. To confirm the relationship between YAP- and EMT-related molecules, we transfected a YAP plasmid into the A549 cell line. Western blot analysis showed that ectopic YAP expression downregulated WWC3 expression and increased expression levels of N-cadherin, Snail, and Slug (Figure 4A). This was consistent with findings on colorectal cancer and hepatocellular carcinoma reported by Ling et al, ${ }^{26}$ Wang et al, ${ }^{27}$ and Jayachandran et al. ${ }^{28}$ 
Table 3 Correlation of WWC3 with the expression of Snail and Slug in 127 NSCLC specimens

\begin{tabular}{|c|c|c|c|c|}
\hline \multirow[t]{2}{*}{ EMT-markers } & \multicolumn{2}{|l|}{ WWC3 } & \multirow[t]{2}{*}{$\chi^{2}$} & \multirow{2}{*}{$\begin{array}{l}P \text {-value } \\
\text { (two sided) }\end{array}$} \\
\hline & Negative & $\overline{\text { Positive }}$ & & \\
\hline Snail & & & 10.863 & $0.001 *$ \\
\hline Negative & 26 & 37 & & \\
\hline Positive & 45 & 19 & & \\
\hline Slug & & & 6.842 & $0.012 *$ \\
\hline Negative & 33 & 39 & & \\
\hline Positive & 38 & 17 & & \\
\hline
\end{tabular}

Note: *Significant difference.

Abbreviations: WWC3, WW and C2 domain containing protein-3; NSCLC, nonsmall-cell lung cancer.

Western blot analysis demonstrated that WT WWC3 plasmid could upregulate the phosphorylation levels of LATS1 and YAP, the mutant lacking a WW domain, which was unable to interact with LATS1 and abrogated this role (Figure 4B). Next, we cotransfected shRNA-WWC3 and siRNA-LATS1 into A549 cells, and immunoblotting revealed that a LATS1 knockdown could reverse the WWC3-induced changes to the EMT-related molecules (Figure 4C). In addition, shRNAWWC3 and shYAP were cotransfected into A549 cells, and the results also indicated that silencing YAP could reverse the EMT molecular changes caused by the WWC3 knockdown (Figure 4D). To determine whether WWC3 negatively regulates EMT processes by stimulating the Hippo pathway, VP, a YAP-TEAD interaction inhibitor, was also recognized as a Hippo pathway inhibitor. A Western blot analysis demonstrated that VP can reverse the changes to E-cadherin, N-cadherin, Snail, and Slug that were induced by WWC3 silencing, as do LATS1 and YAP knockdowns (Figure 4E). YAP knockdown also abrogated the enhanced migration and invasive abilities of lung cancer cells that were caused by the WWC3 knockdown (Figure 4F). Based on these results, we concluded that WWC3 inhibits EMT processes by stimulating the Hippo pathway.

\section{Discussion}

Lung cancer is an aggressive cancer composed of large amounts of functionally and phenotypically distinct cells. ${ }^{1}$ During cancer progression, neoplastic cells can undergo EMT processes and acquire stem-like traits, leading to metastatic dissemination, chemoresistance, and poor prognosis, such as in liver cancer. ${ }^{28}$ The mechanisms that cause the malignant transition and cancer remain unclear. In this study, we demonstrated that downregulating WWC3 induces EMT features and enhances the migratory and invasive abilities of lung cancer cells. Combined with our previous findings that WWC3 expression was low in lung cancer cell lines and tissues, we assumed that WWC3 downregulation may be one of the primary causes of bronchial epithelial cell transition. However, the reasons for the low WWC3 expression in lung cancer, for example, methylation, gene mutation, and tissue specificity, need further investigation.

In recent decades, the correlation between the Hippo pathway and the EMT process has garnered much attention. Inactivating the Hippo pathway is correlated with the EMT process. Expression of YAP correlates with EMT marker expression and enhanced tumor migration, invasion, chemotherapy resistance, and poor prognosis. ${ }^{26,29,30}$ Dysregulation of upstream molecules of the Hippo pathway, such as Willin/FRMD6 and KIBRA, also contributes to inducing the EMT process. We found that ectopic expression of WWC3, also identified as an upstream molecule of the Hippo pathway, increases expression of the EMT-related biomarkers, such as E-cadherin, ZO-1, and occludin, and decreases $\mathrm{N}$-cadherin expression. These results were confirmed by WWC3 gene silencing, which enhanced N-cadherin levels and decreased E-cadherin, ZO-1, and occludin levels. The immunoblotting assay showed that WWC3- $\Delta \mathrm{WW}$ plasmid transfection abrogated this effect as does the WT. Loss of E-cadherin expression is a hallmark of the EMT process, and transcription factors that regulate E-cadherin transcription such as Snail, Slug, and ZEB1 are the focus of investigation. ${ }^{31}$ We transfected a WWC3 WT plasmid and its mutant $(-\Delta \mathrm{WW})$, and the result was that WT WWC3 repressed Snail and Slug expression, but not ZEB1. WWC3- $\triangle \mathrm{WW}$ had no effect. These changes were demonstrated by WWC3 knockdown using two independent shRNAs. The WW domain of WWC3 is key for the association between WWC3 and LATS1. Mutant WWC3 lacking a WW domain is unable to bind to LATS1; therefore, it cannot promote the phosphorylation of LATS1 and subsequently YAP. The importation level of the YAP nucleus then increases, enhancing Snaill and Slug transcription. Recently, Tang et $\mathrm{al}^{24,25}$ reported that Snail and Slug interact with YAP and TAZ in stem cell self-renewal and differentiation and the mesenchymal stem cell function and bone formation. This demonstrates the potential relationship between the Hippo pathway and the EMT process. In our study, we observed that by interfering with LATS1 and YAP, WWC3 knockdown did not influence the EMT process, which was also confirmed by an inhibitor of the YAP-TEAD interaction (VP).

Recently, some researchers reported that YAP and TAZ could regulate $\mathrm{PD}-\mathrm{L} 1$ expression in lung cancer cells; ${ }^{32,33}$ Ghiso et $\mathrm{al}^{34}$ reported that YAP can also mediate resistance to 
A

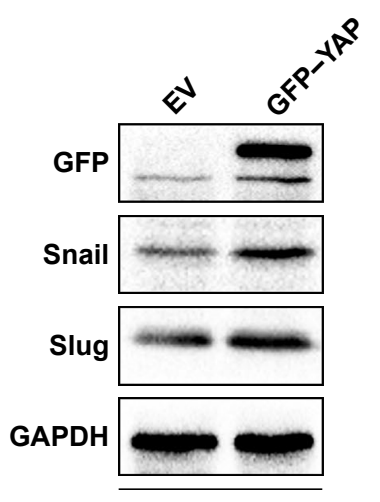

D

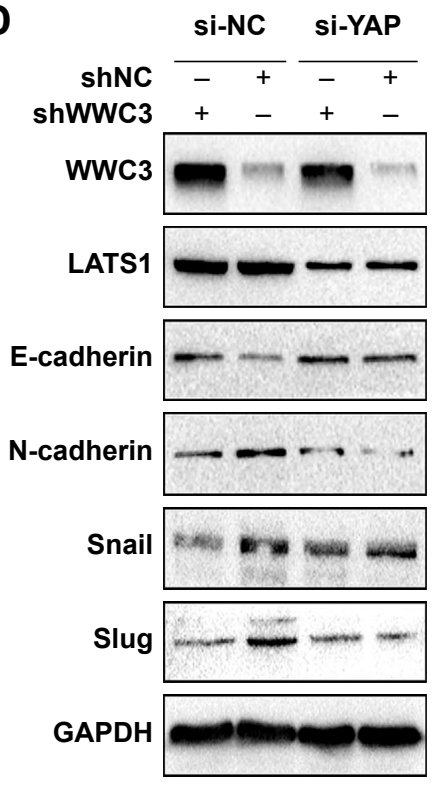

B

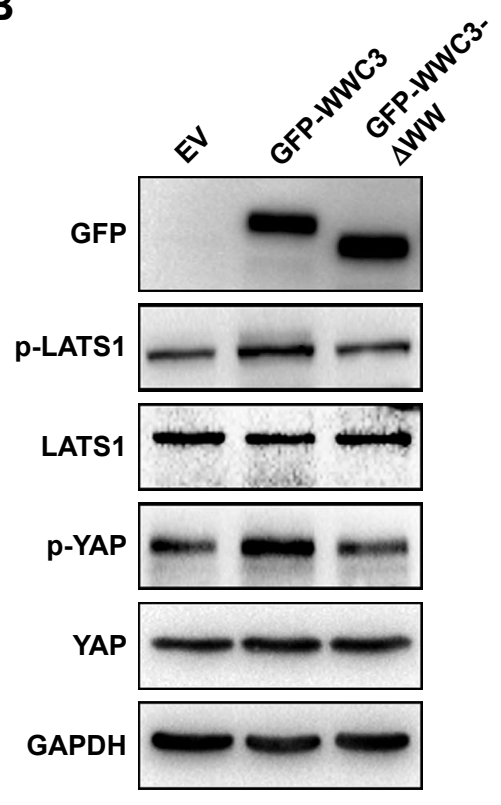

E

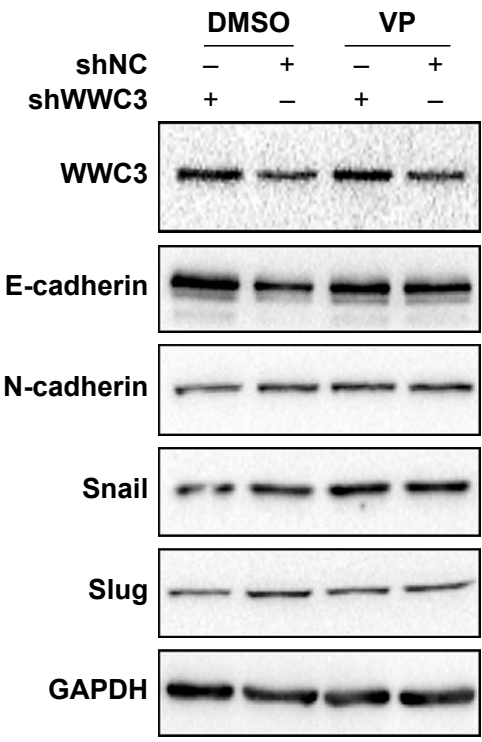

C

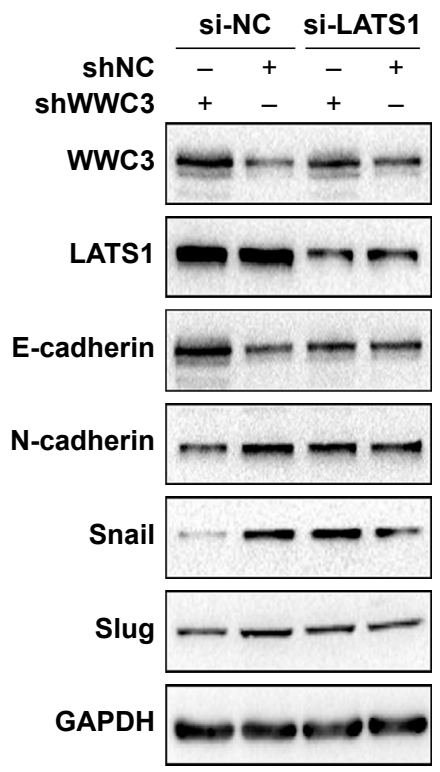

F
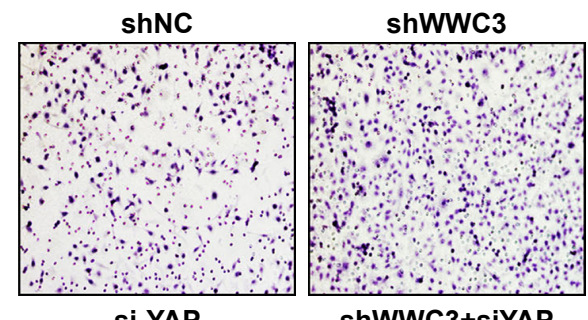

si-YAP

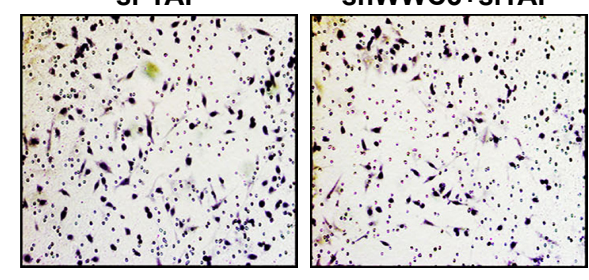

A549

Figure 4 WWC3 activates the Hippo pathway via LATSI, suppressing the EMT process.

Notes: (A) YAP transfection in HI299 upregulates Snail and Slug expression. GAPDH was used as a loading control. (B) WT WWC3 upregulated Hippo-related key molecules, the phosphorylation levels of LATSI, and YAP, whereas mutant could not. WT or mutant plasmid was transfected into HI 299 cells, and subsequently, the proteins were analyzed by immunoblotting. Total LATSI and YAP were used as loading controls. (C and D) LATSI or YAP knockdown reversed the changes of EMT caused by WWC3 silencing. A549 cells were transfected with shCTL, shWWC3, and siRNA-LATSI/siRNA-YAP as indicated, lysed, and analyzed by immunoblotting. (E) VP reversed the changes of EMT caused by WWC3 silencing. A549 cells were transfected with shRNA-WWC3 or control vector, and $36 \mathrm{~h}$ later, the YAP-TEAD interaction inhibitor VP was treated for $12 \mathrm{~h}$. Cell lysates were then analyzed by immunoblotting with the indicated antibodies. (F) YAP silencing reversed the enhanced invasive ability of lung cancer cells caused by WWC3 knockdown. Boyden chamber transwell assay of cellular invasion and the mean number of cells in five fields per membrane (A549, shWWC3 vs control, number: $213 \pm 14$ vs $583 \pm 17, P<0.001$; siRNA-YAP vs shWWC3+siRNA-YAP, number: $95 \pm 10$ vs $85 \pm 5, P>0.05$ ). The magnification is $400 \times$.

Abbreviations: WWC3, WW and C2 domain containing protein-3; LATSI, large tumor suppressor-I; EMT, epithelial-mesenchymal transition; YAP, yes-associated protein; GAPDH, glyceraldehyde-3-phosphate dehydrogenase; WT, wild type; shCTL, short-hairpin RNA control; VP, verteporfin; TEAD, TEA domain transcription factors; EV, empty vector.

EGFR inhibitors in NSCLC. These results implied the close relevance between Hippo pathway and TKIs or PD-1 inhibitors therapy. In our previous work, we demonstrated that WWC3 overexpression attenuates YAP nuclear localization.
Hence, we hypothesize that WWC3 could serve as a new therapeutic target combined with TKIs or PD1/PD-L1 and probably may prolong survival of patients, but this needs further more investigation. 


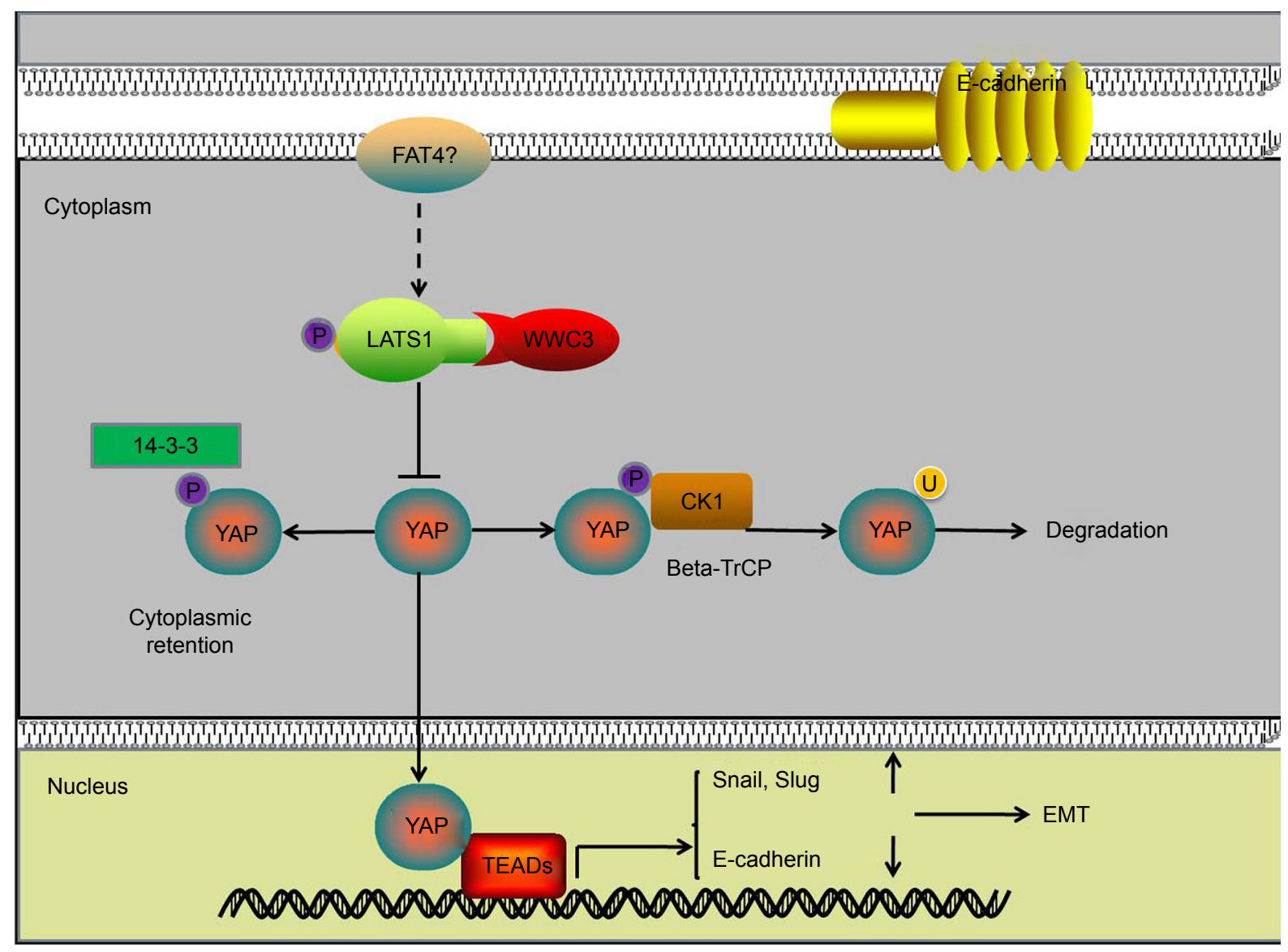

Figure 5 Pattern diagram showing the impact of WWC3-mediated regulation of Hippo signaling on the EMT process.

Abbreviations: WWC3, WW and C2 domain containing protein-3; EMT, epithelial-mesenchymal transition; LATSI, large tumor suppressor- I; YAP, yes-associated protein; TEAD, TEA domain transcription factors.

\section{Conclusion}

Downregulating WWC3 in lung cancer cells decreases LATS1 interaction, attenuates YAP phosphorylation, and inhibits the Hippo pathway, thus promoting the EMT process and the migration and invasion of lung cancer (Figure 5).

\section{Acknowledgment}

This study was supported by the National Natural Science Foundation of China (grant 81572854 to Enhua Wang, grant 81401885 to Xuyong Lin, and grant 81672302 to Di Zhang.

\section{Author contributions}

All authors contributed toward data analysis, drafting and revising the paper and agree to be accountable for all aspects of the work.

\section{Disclosure}

The authors report no conflicts of interest in this work.

\section{References}

1. Travis WD. Pathology of lung cancer. Clin Chest Med. 2002;23(1): 65-81, viii.

2. Jemal A, Siegel R, Ward E, Hao Y, Xu J, Thun MJ. Cancer statistics. CA Cancer J Clin. 2009;59(4):225-249.

3. Minna JD, Roth JA, Gazdar AF. Focus on lung cancer. Cancer Cell. 2002;1(1):49-52

4. Schiller JH, Harrington D, Belani CP, et al; Eastern Cooperative Oncology Group. Comparison of four chemotherapy regimens for advanced nonsmall-cell lung cancer. N Engl J Med. 2002;346(2):92-98.

5. Wang S, Song Y, Yan F, Liu D. Mechanisms of resistance to thirdgeneration EGFR tyrosine kinase inhibitors. Front Med. 2016;10(4): 383-388.

6. Ma W, Gilligan BM, Yuan J, Li T. Current status and perspectives in translational biomarker research for PD-1/PD-L1 immune checkpoint blockade therapy. J Hematol Oncol. 2016;9(1):47.

7. Wang S, Cang S, Liu D. Third-generation inhibitors targeting EGFR T790M mutation in advanced non-small cell lung cancer. $J$ Hematol Oncol. 2016;9:34.

8. O'Mahony D, Kummar S, Gutierrez ME. Non-small-cell lung cancer vaccine therapy: a concise review. J Clin Oncol. 2005;23(35): 9022-9028.

9. Shepherd FA, Rodrigues Pereira J, Ciuleanu T, et al; National Cancer Institute of Canada Clinical Trials Group. Erlotinib in previously treated non-small-cell lung cancer. $N$ Engl J Med. 2005;353(2):123-132. 
10. Acloque H, Adams MS, Fishwick K, Bronner-Fraser M, Nieto MA. Epithelial-mesenchymal transitions: the importance of changing cell state in development and disease. J Clin Invest. 2009;119(6):1438-1449.

11. Papageorgis P. TGF- $\beta$ signaling in tumor initiation, epithelial-tomesenchymal transition, and metastasis. J Oncol. 2015;2015:587193.

12. Gonzalez DM, Medici D. Signaling mechanisms of the epithelialmesenchymal transition. Sci Signal. 2014;7(344):re8.

13. Harvey KF, Zhang X, Thomas DM. The hippo pathway and human cancer. Nat Rev Cancer. 2013;13:246-257.

14. Wennmann DO, Schmitz J, Wehr MC, et al. Evolutionary and molecular facts link the WWC protein family to hippo signaling. Mol Biol Evol. 2014;31(7):1710-1723.

15. Dasari VR, Mazack V, Feng W, Nash J, Carey DJ, Gogoi R. Verteporfin exhibits YAP-independent anti-proliferative and cytotoxic effects in endometrial cancer cells. Oncotarget. 2017;8(17):28628-28640.

16. Han Q, Lin X, Zhang X, et al. WWC3 regulates the Wnt and hippo pathways via dishevelled proteins and large tumour suppressor 1, to suppress lung cancer invasion and metastasis. J Pathol. 2017;242(4): 435-447.

17. Hou J, Zhou J. WWC3 downregulation correlates with poor prognosis and inhibition of Hippo signaling in human gastric cancer. Onco Targets Ther. 2017;10:2931-2942.

18. Travis WD, Brambilla E, Burke AP, et al. The 2015 World Health Organization classification of lung tumors: impact of genetic, clinical and radiologic advances since the 2004 classification. J Thorac Oncol. 2015;10(9):1243-1260.

19. Goldstraw P. Updated staging system for lung cancer. Surg Oncol Clin N Am. 2011;20(4):655-666.

20. Lin XY, Zhang XP, Wu JH, Qiu XS, Wang EH. Expression of LATS1 contributes to good prognosis and can negatively regulate YAP oncoprotein in non-small-cell lung cancer. Tumor Biol. 2014;35(7): 6435-6443.

21. Miao Y, Li AL, Wang L, et al. Expression of p130cas, E-cadherin and $\beta$-catenin and their correlation with clinicopathological parameters in non-small cell lung cancer: p130cas over-expression predicts poor prognosis. Folia Histochem Cytobiol. 2012;50(3):392-397.

22. Shi L, Wang Y, Lu Z, Zhang H, Zhuang N, Wang B. miR-127 promotes EMT and stem-like traits in lung cancer through a feed-forward regulatory-loop. Oncogene. 2017;36(12):1631-1643.
23. Zhang X, Liu X, Luo J, et al. Notch3 inhibits epithelial-mesenchymal transition by activating Kibra-mediated Hippo/YAP signaling in breast cancer epithelial cells. Oncogenesis. 2016;5(11):e269.

24. Tang Y, Feinberg T, Keller ET, Li XY, Weiss SJ. Snail/Slug binding interactions with YAP/TAZ control skeletal stem cell self-renewal and differentiation. Nat Cell Biol. 2016;18(9):917-929.

25. Tang Y, Weiss SJ. Snail/Slug-YAP/TAZ complexes cooperatively regulate mesenchymal stem cell function and bone formation. Cell Cycle. 2017;16(5):399-405.

26. Ling HH, Kuo CC, Lin BX, Huang YH, Lin CW. Elevation of YAP promotes the epithelial-mesenchymal transition and tumor aggressiveness in colorectal cancer. Exp Cell Res. 2017;350(1):218-225.

27. Wang S, Li H, Wang G, et al. Yes-associated protein (YAP) expression is involved in epithelial-mesenchymal transition in hepatocellular carcinoma. Clin Transl Oncol. 2016;18(2):172-177.

28. Jayachandran A, Dhungel B, Steel JC. Epithelial-to-mesenchymal plasticity of cancer stem cells: therapeutic target in hepatocellular carcinoma. J Hematol Oncol. 2016;9(1):74.

29. McGowan M, Kleinberg L, Halvorsen AR, Helland Å, Brustugun OT. NSCLC depend upon YAP expression and nuclear localization after acquiring resistance to EGFR inhibitors. Genes Cancer. 2017;8(3-4): 497-504.

30. Yuan Y, Li D, Li H, Wang L, Tian G, Dong Y. YAP overexpression promotes the epithelial-mesenchymal transition and chemoresistance in pancreatic cancer cells. Mol Med Rep. 2016;13(1):237-242.

31. Dong T, Zhang Y, Chen Y, et al. FOXO1 inhibits the invasion and metastasis of hepatocellular carcinoma by reversing ZEB2-induced epithelialmesenchymal transition. Oncotarget. 2017;8(1):1703-1713.

32. Miao J, Hsu PC, Yang YL, et al. YAP regulates PD-L1 expression in human NSCLC cells. Oncotarget. 2017;8(70):114576-114587.

33. Janse RHJ, Azad T, Ling M, et al. The hippo pathway components TAZ promotes immune evasion in human cancer through PD-L1. Cancer Res. 2018;78(6):1457-1470.

34. Ghiso E, Migliore C, Ciciriello V, et al. YAP-dependent AXL overexpression mediates resistance to EGFR inhibitors in NSCLC. Neoplasia. 2017;19(12):1012-1021.
OncoTargets and Therapy

\section{Publish your work in this journal}

OncoTargets and Therapy is an international, peer-reviewed, open access journal focusing on the pathological basis of all cancers, potential targets for therapy and treatment protocols employed to improve the management of cancer patients. The journal also focuses on the impact of management programs and new therapeutic agents and protocols on

\section{Dovepress}

patient perspectives such as quality of life, adherence and satisfaction. The manuscript management system is completely online and includes a very quick and fair peer-review system, which is all easy to use. Visit http://www.dovepress.com/testimonials.php to read real quotes from published authors. 